Results Compared to monotherapies, combined Gvax/ $\alpha \mathrm{CD} 25$ significantly delayed tumour growth and prolonged survival, in association with enhanced infiltration of $\mathrm{T}$ cells with an activated phenotype. Approximately $50 \%$ of mice achieved partial response with relapse at day 35-45 post tumor injection. To characterize immune evolution prior to relapse, we analysed stable, partially responding tumors and paired draining lymph nodes (DLNs). Over time, activated PD-1 +ICOS+TCF7- T cells with an effector memory (CD44 +CD62L-) phenotype fell from $30 \%$ to $10 \%$ whilst resting, TCF7 + early differentiated cells rose in abundance towards levels seen in untreated tumors. Abundance of Ki67-, resting Tregs also recovered. Similar results were obtained in analysis of DLNs.

Conclusions Combined Treg depletion/whole tumor vaccination therapy is effective in a poorly infiltrated B16 melanoma model. Combined treatment promotes $\mathrm{T}$ cell infiltration and activation. In mice achieving a partial response, treatment effects on the immune landscape were observed to decay over time suggesting a return to immune equilibrium. Further studies to explore the mechanistic basis of this observation are underway.

Disclosure Information C. Qing: None. E. Ghorani: None. I. Solomon: None. F. Gálvez-Cancino: None. F. Vargas: None. K. Peggs: None. S. Quezada: B. Research Grant (principal investigator, collaborator or consultant and pending grants as well as grants already received); Significant; F. HOFFMANNLA ROCHE LTD.

\section{P03.24 CALRETICULIN EXPOSURE ON MALIGNANT BLASTS CORRELATES WITH IMPROVED NK CELL-MEDIATED CYTOTOXICITY IN AML PATIENTS}

${ }^{1}$ T Truxova, ${ }^{1,2} \mathrm{~L}$ Kasikova, ${ }^{3,4} \mathrm{C}$ Salek, ${ }^{1} \mathrm{M}$ Hensler, ${ }^{5} \mathrm{D}$ Lysak, ${ }^{1,2} \mathrm{P}$ Holicek* $^{*}$, $\mathrm{P}$ Bilkova, ${ }^{6} \mathrm{M}$ Holubova, ${ }^{7} \mathrm{X}$ Chen, ${ }^{8} \mathrm{R}$ Mikyskova, ${ }^{8} \mathrm{M}$ Reinis, ${ }^{9} \mathrm{M}$ Kovar, ${ }^{9} \mathrm{~B}$ Tomalova, $7,10,11 \mathrm{JP}$ Kline, 12,13,14,15 L Galluzzi, 1,2R Spisek, 1,2J Fucikova. 'Sotio a.S, Prague, Czech Republic; ${ }^{2}$ Department of Immunology, Charles University, 2nd Faculty of Medicine and University Hospital Motol, Prague, Czech Republic; ${ }^{3}$ Institute of Hematology and Blood Transfusion, Prague, Dominican Republic, ${ }^{4}$ Institute of Clinical and Experimental Hematology, 1 st Faculty of Medicine, Charles University, Prague, Czech Republic; ${ }^{5}$ Department of Hematology and Oncology, University Hospital in Pilsen, Pilsen, Czech Republic; ${ }^{6}$ Laboratory of Tumor Biology and Immunotherapy, Biomedical Center, Faculty of Medicine in Pilsen, Charles University, Pilsen, Czech Republic; ' Department of Medicine, University of Chicago, Chicago, IL, USA; ${ }^{8}$ Laboratory of Immunological and Tumour models, Institute of Molecular Genetics of the Czech Academy of Sciences, Prague, Czech Republic; ${ }^{9}$ Laboratory of Tumor Immunology, Institute of Microbiology of the Czech Academy of Sciences, Prague, Czech Republic; ${ }^{10}$ Committee on Immunology, University of Chicago, Chicago, Chicago, IL, USA; ${ }^{11}$ University of Chicago Comprehensive Cancer Center, Chicago, IL, USA; ${ }^{12}$ Department of Radiation Oncology, Weill Cornell Medical College, New York, NY, USA; ${ }^{13}$ Sandra and Edward Meyer Cancer Center, New York, NY, USA; ${ }^{14}$ Caryl and Israel Englander Institute for Precision Medicine, New York, NY, USA; ${ }^{15}$ Department of Dermatology, Yale School of Medicine, New Haven, CT, USA

\subsection{6/jitc-2020-ITOC7.62}

In some settings, cancer cells responding to treatment undergo an immunogenic form of cell death that is associated with the abundant emission of danger signals in the form of damage-associated molecular patterns. Accumulating preclinical and clinical evidence indicates that danger signals play a crucial role in the (re-)activation of antitumor immune responses in vivo, thus having a major impact on patient prognosis. We have previously demonstrated that the presence of calreticulin on the surface of malignant blasts is a positive prognostic biomarker for patients with acute myeloid leukemia (AML). Calreticulin exposure not only correlated with enhanced T-cell-dependent antitumor immunity in this setting but also affected the number of circulating natural killer (NK) cells upon restoration of normal hematopoiesis. Here, we report that calreticulin exposure on malignant blasts is associated with enhanced NK cell cytotoxic and secretory functions, both in AML patients and in vivo in mice. The ability of calreticulin to stimulate NK-cells relies on CD11c+CD14high cells that, upon exposure to CRT, express higher levels of IL-15R $\alpha$, maturation markers (CD86 and HLA- DR) and CCR7. CRT exposure on malignant blasts also correlates with the upregulation of genes coding for type I interferon. This suggests that CD11c+CD14high cells have increased capacity to migrate to secondary lymphoid organs, where can efficiently deliver stimulatory signals (IL-15R $\alpha / \mathrm{IL}-15)$ to NK cells. These findings delineate a multipronged, clinically relevant mechanism whereby surfaceexposed calreticulin favors NK-cell activation in AML patients.

Disclosure Information I. Truxova: None. L. Kasikova: None. C. Salek: None. M. Hensler: None. D. Lysak: None. P. Holicek: None. P. Bilkova: None. M. Holubova: None. X. Chen: None. R. Mikyskova: None. M. Reinis: None. M. Kovar: None. B. Tomalova: None. J.P. Kline: None. L. Galluzzi: None. R. Spisek: None. J. Fucikova: None.

\section{P03.25 NEUTRALIZING EXTRACELLULAR CHP-1 IMPAIRS TUMOR GROWTH AND METASTASIS FORMATION}

L Secli* F Fusella, M Brancaccio. University of Turin, Torino, Italy

\subsection{6/jitc-2020-ITOC7.63}

Background Found in the extracellular compartment, Heat Shock Proteins (HSPs) are actively secreted proteins that modulate the tumor behavior. Extracellular HSPs play a unique role as extracellular chaperones and receptors-binding molecules, favoring the establishment and maintenance of different cancer hallmarks, including immune modulation and evasion. CHP-1, is a ubiquitously expressed protein with chaperone activity and its high expression correlates with high tumor grade and lymph node positivity in different breast and lung cancer subtypes. In addition, CHP-1 is actively and uncanonically secreted by cancer cells in the tumor microenvironment (TME).

Materials and Methods Sera cancer patients were analyzed for the presence of CHP-1. To assess the role of extracellular CHP-1 (eCHP-1) in the TME, in vitro experiments on different cell populations have been performed. To dissect the molecular mechanisms, through which eCHP-1 induces cancer progression, have been analyzed specific signaling pathways in cancer and immune cells. Immune cell composition in presence of eCHP-1 in tumors has been identified using flowcytometry. The characterization of eCHP-1 inhibition as therapeutic approach has been conducted in breast and colon cancer pre-clinical models.

Results eCHP-1 activates an autocrine signaling through TLR2, TLR4 and LRP1, promoting tumor progression and metastasis formation in different pre-clinical models. Moreover, eCHP-1 can modulate the immune composition of the TME, making interesting the analysis of its inhibition in cancer immunotherapy. 
Conclusions eCHP-1 represents a easy accessible protein for diagnosis and targeting in very aggressive canncers.

Disclosure Information L. Seclì: None. F. Fusella: None. M. Brancaccio: None.

\section{P03.26 IMMUNOPROFILING OF ORAL AND OROPHARYNGEAL TUMORS OF DIFFERENT ETIOLOGY}

${ }^{1}$ B Pokrýuková*, ${ }^{2} \mathrm{M}$ Grega, ${ }^{3} \mathrm{~J}$ Klozar, ${ }^{4} \mathrm{O}$ Vencalek, ${ }^{1} \mathrm{~J}$ Nunvar, ${ }^{1} \mathrm{R}$ Tachezy. ${ }^{1}$ Charles University, Faculty of Science, BIOCEV, Vestec, Czech Republic; ${ }^{2}$ Charles University, Department of Pathology and Molecular Medicine, 2nd Faculty of Medicine, Prague, Czech Republic; ${ }^{3}$ Charles University, University Hospital Motol, Department of Otorhinolaryngology and Head and Neck Surgery, 1st Faculty of Medicine, Prague, Czech Republic; ${ }^{4}$ Faculty of Science of the Palacky University in Olomouc, Department of Mathematical Analysis and Applications of Mathematics, Olomouc, Czech Republic

\subsection{6/jitc-2020-ITOC7.64}

Background Head and neck carcinomas (HNC) are the world's sixth most common cancer. Most of HNCs are associated with tobacco and other environmental factors but a growing part of oropharyngeal tumors are caused by persistent infection of human papillomavirus (HPV). Patients with HPV positive cancers have a better prognosis with fewer recurrences. This may be caused by different anti-tumor immune response and immune profile of patients. Multispectral fluorescent immunohistochemistry (fIHC) is a powerful tool for a detailed analysis of the tumor microenvironment. This method allows to access the phenotype and calculate cells in tumor parenchyma and stroma of the tumor since in comparison to flow cytometry, an architecture of the tissue remains preserved. fIHC is uniquely suited to study interaction of immune and cancer cells in situ.

Materials and Methods Number of 97 formalin fixed paraffine-embedded slides of the human HNC tissue with known etiology were examined using 4 different panels of 5 antibodies each. These panels include antibodies suitable for phenotyping of immune cells (CD3e, CD4, CD8, FOXP3) or their functional description (PD1, CTLA4, ICOS, CCR4). Additionally, antibodies against Ki67, VEGF and cell cytokeratin were used. Slides were stained using Opal ${ }^{\mathrm{TM}}$ 7-Color Fluorescent IHC Kit (Akoya Biosciences). The quantity of immune cells was evaluated in stroma and tumor compartment using InForm $^{\mathrm{TM}}$ 2.4.6. software (Akoya Biosciences). For all patients the demographic and clinical data were available and these patients were followed for up to 18 years.

Results Our results have shown significantly higher abundance of Th and Tc in both compartments of HPV+ samples. Besides HPV etiology Th and Tc in the tumor microenvironment predict independently better survival of patients. We did not observed difference in number of Tregs (characterized as a CD3+CD4+FOXP3 + cells) in tumors of different etiology, but we detected higher number of ICOS+Tregs in stroma of HPV- tumors. We also quantified the subpopulations of Th and Tc cells expressing regulatory receptors PD1 and CTLA4. PD1 showed significantly higher expression on $\mathrm{Th}$ and $\mathrm{Tc}$ both in tumor and stroma of HPV+ tumors, but CTLA4 expression was significantly higher only on Th located in stroma of HPV- tumors. Moreover, we detected significantly higher VEGF expression in both compartments and higher proliferating activity of tumor cells in HPV- tumors.

Conclusions Detailed analyses of the tumor infiltrating lymphocytes allows for selection of prognostic markers in HNC of different etiology. Our results may also help to understand the better prognosis of HPV+ patients. More detailed survival analyses with inclusion of other clinical and demographic data will be presented.

Disclosure Information B. Pokrývková: None. M. Grega: None. J. Klozar: None. O. Vencalek: None. J. Nunvar: None. R. Tachezy: None.

\section{P03.27 ROLE OF NOX2 FOR HYPOXIA-INDUCED CHEMORESISTANCE IN ACUTE MYELOID LEUKEMIA}

S Paul*, H Grauers Wiktorin, R Kiffin, K Hellstrand, A Martner. TIMM Laboratory, Sahlgrenska Cancer Center, Institute of Biomedicine, Sahlgrenska Academy, University of Gothenburg, Gothenburg, Sweden

\subsection{6/jitc-2020-ITOC7.65}

Background Relapse of acute myeloid leukemia (AML) may arise from residual chemoresistant leukemic cells. A hypoxic tumor microenvironment, such as the bone marrow, is known to enhance chemoresistance in various forms of cancer, including AML. Hypoxia inducible factor 1 alpha (HIF-1 $\alpha)$ is an important mediator of cellular adaptation to hypoxia. HIF- $1 \alpha$ is a constitutively expressed transcription factor that is rapidly degraded under normoxic conditions after hydroxylation by oxygen sensors, such as the HIF prolyl hydroxylases (PHDs). However, under hypoxic conditions the oxygen sensors lose the ability to induce the degradation of HIF- $1 \alpha$ resulting in its stabilization and translocation to the nucleolus where it induces the transcription of genes associated with glucose metabolism, angiogenesis, and cell survival. This may result in proliferation of malignant cells, impaired tumor cell differentiation and chemoresistance. Reactive oxygen species (ROS) have been shown to inhibit PHDs and may thereby stabilize HIF-1 $\alpha$, and may thus contribute to chemoresistance. AML cells may generate ROS via the myeloid NADPH oxidase NOX2. We therefore hypothesized that NOX inhibitors would decrease chemoresistance in a hypoxic environment.

Materials and Methods The wild type (WT) AML cell line PLB-985 and its NOX2 knocked out (KO) counterpart were cultured for five days in hypoxia ( $1 \%$ oxygen) or normoxia (21\% oxygen) in the presence or absence of the NOX inhibitors histamine dihydrochloride (HDC), diphenyleneiodonium (DPI) and GSK2795039. Thereafter cells were exposed to the chemotherapeutic agent daunorubicin for 48 hours (in hypoxia or normoxia) and cell death was determined using the XTT assay. Stabilization of HIF- $1 \alpha$ was measured either by western blot or flow cytometry. Differentiation of cells was quantified by measuring the expression of CD14 and CD11b by flow cytometry.

Results Hypoxia reduced the sensitivity of WT PLB-985 cells to daunorubicin induced cell death $(P<0.05, \mathrm{n}=4)$ whereas NOX2 KO cells were equally sensitive to daunorubicin in hypoxia and normoxia $(P>0.5, \mathrm{n}=4)$. Furthermore, NOX2 KO AML cells displayed increased sensitivity to daunorubicin induced killing compared with PLB WT cells in a hypoxic environment $(P<0.05, \mathrm{n}=4)$. Preliminary results show that pharmacological NOX inhibition using DPI enhanced the sensitivity of WT AML cells to daunorubicin induced killing. These results suggests that functional NOX2 contributes to chemoresistance in a hypoxic environment. As expected, hypoxia stabilized the expression of HIF- $1 \alpha$ in AML cells. Preliminary results suggest that HIF- $1 \alpha$ expression was reduced in the presence of NOX inhibitors. 